\title{
Integrated comparative genomoc analysis and phenotypic profiling of Pseudomonad aeruginosa isolates from crude oil
}

Anming Xu1, 2\#, Di Wangl\#, Yichen Ding, Yaqian Zheng1, 2, Bo Wang1,2, Qing Wei1, Shiwei Wang1, Liang Yang, Luyan Z Ma1, 2*

1 Institute of Microbiology, Chinese Academy of Sciences, Beijing 100101, China, *email: Luyanma27@im.ac.cn ${ }_{2}$ University of Chinese Academy of Sciences, Beijing 100049, China

3 Nanyang Technological University, Singapore

4Southern University of Science and Technology, Shenzhen 518055, China

Pseudomonas aeruginosa is an environmental microorganism that can thrive in diverse ecological niches including plants, animals, water, soil, and crude oil. It is also one of microorganisms widely used in tertiary petroleum recovery and decontamination of crude oil pollution. However, the genomic information regarding the mechanism of survival and superiority of this bacteria in crude oil is still limited. In this study, three Pseudomonads strains (named as IMP66, IMP67 and IMP68) isolated from crude oil were taken for whole-genome sequencing analysis by a hybridized PacBio and Illumina approach. The phylogeny analysis based on 10 housekeeping genes showed that all three strains clustered together with $P$. aeruginosa species. Subsequent comparative genomic analysis revealed a high degree of individual genomic plasticity, with a probable alkane degradation genomic island, one type I-F CRISPR-Cas system and several prophages were integrated into their genomes compared to the laboratory strain PAO1. Nine genes encoding alkane hydroxylases (AHs) homologues were found in each strain. This was consistent with their ability to live in the crude oil environment. $P$. aeruginosa can produce rhamnolipids (RLs) biosurfactant to emulsify oil. Analysis of quorum sensing (QS) signal molecules that regulating rhamnolipids synthesis showed that IMP67 and IMP68 produced more $\mathrm{N}$-acylhomoserine lactones (AHLs) signal molecules than that of PAO1, which was in agreement with their high RLs yield reported previously. Acquiring alkane-degradation ability and the enhanced-expression of QS-regulated gene might enable $P$. aeruginosa to adapt in crude oil environment. This study provided the genetic evidence for adaptation of $P$. aeruginosa in crude oil and bridged the potential applications of IMP67 and IMP68 in microbial-enhanced oil recovery and bioremediation. 